\title{
Simulation Framework of Caching Techniques for Adoptability of M-Commerce in Location based Services
}

\author{
Archana Sharma \\ Research Scholar \\ Mewar University,Rajasthan
}

\author{
Vineet Kansal, Ph.D \\ Director IT \\ ITS Engineering College,Greater Noida
}

\author{
RPS. Tomar, Ph.D \\ Professor-MCA Dept \\ IPEM,Ghaziabad
}

\begin{abstract}
Mobile commerce enables access to tangible \& intangible products and services regardless of the location of either buyer or seller, in many situations the specific location of the buyer and seller is critical to the transaction. A host of new location-based applications and services are emerging with significant implications for the future of m-commerce. One of the main goals of mobile data access is to reach the ubiquity inherent to the mobile systems: to access information regardless of time and place. Data replication not only ensure the availability of the data but also reduce the communication cost, increase data sharing and increase the safety of the critical data. Furthermore, it also determine when and where (location) to place the replica of data, controlling the number of data replicas over a network for efficient utilization of the network resources. In mobile database systems caching proved itself as an important technique to optimize the way a mobile database is used. In order to improve data availability on the DBMS hosted on mobile device side, this paper develop a Framework through simulation study to analyze the communication cost involved by different caching technique which selects a priori subset of the data available on the server side database and copy them to the mobile device side Database.
\end{abstract}

Keywords: Mobility, Pre-fectching, Location-Based Services,

\section{INTRODUCTION}

The importance of location in m-commerce has been linked to the relative success of click and mortar m-commerce business models compared to digital pure plays[1]. Clearly for mcommerce, there are many types of services, such as those providing navigation, directory search, and ticketing/permission for entry, where the location of the consumer is of prime importance. Hence, localization and personalization are additional dimensions of $\mathrm{m}$-commerce value creation [2]. It classify $\mathrm{m}$ - commerce services into two broad categories: those that are requested by users once their location is determined, and those that are triggered automatically once a certain condition is met (e.g. a boundary is crossed)[3]. In addition, five groups of application areas are identified: communication, fleet management, routing, safety and security, and entertainment [3]. Further it can also be classified according to whether they apply to consumers, business customers, or employees in a firm. Besides this cellular operators are beginning to offer different rates based on the location of callers. M-commerce services might include use of the wireless device to make payments for tickets at theaters and on public transportation, vending machines, and for goods in shops to speed up checkouts. Often this capability requires that wireless devices exchange payment information with local POS devices[4].
This paper highlights the three challenges in mobile transactions, i.e frequent disconnection, battery consumption and asymmetric communication between mobile client and server which affects the communication cost. The communication between the client and server ought to be minimized to reduce the contentions keeping in view the narrow bandwidth of wireless channels. Data replication and caching are the effectual techniques to efficiently deal with data access to reduce communication cost. Data replication technique increases the availability, scalability and performance in the databases [5]. Moreover,[6] proposes a caching policy and broadcast scheme in which the geographical adjacency and characteristics of target area in Location Dependent Queries (LDQ) are reflected. By applying the moving distance of mobile client, [6] develop the caching policy suitable for urban area. The expectation is: when executing LDQ in local cache, the caching policy offers more accurate answers and significantly improves the workload of mobile clients[7]. Thus an efficient mobile database framework with efficient data replication and caching method is required in order to reduce the communication cost and to improve the performance in a mobile commerce location based services. A common goal in mobile database systems is to minimize the amount of data transmitted over the wireless link between the server and the mobile client [8].

This paper also propose a simulation framework for the mobile database for $\mathrm{m}$ - commerce location based applications to analyze the various caching technique based on certain parameters like access probability, mobility and Hit Ratio . It consists of the central database and location based local databases which are content aware and a partial replication of the central database to the local database. The prime objective of the replication is to ensure the easy availability of data in case of any failure or disastrous event. The data replicated at different local database server will also increase the speed of access and hence reduce the communication cost of the network. Besides this client-intended information in the local database are cached to the client device[9].

\section{MOBILE DATABASE CACHE APPROACHES}

Conventional database caching designs assume both strong connectivity [9] and symmetric bandwidth. In these systems, caching is used primarily as a performance optimization. In mobile environments, where network disconnection and asymmetric bandwidth is a standard mode of operation, caching is essential to maintain service. Caching and replication generally attempt to guarantee that most data requests are for data that is being held in main memory or local storage, negating the need to perform $\mathrm{I} / \mathrm{O}$, or a remote data retrieval. Hence, the use of appropriate caching/replication schemes has been traditionally used to improve performance and reduce service time. In mobile 
environments the performance considerations go beyond simple speedups and data retrieval delays.

\subsection{Pre - fetching}

The mobile devices rely on wireless networks, and due to low bandwidth, high latency, and intermittent connectivity on this network, the interactive response time of on- demand use of unreliable wireless network can be improved by pre-fetching and caching data.

A pre-fetching system predicts the data at the local server, such as an application, will request and hypothetically retrieves and caches that expected data of future need. If the prediction is correct, the cached data is provided on request this improves performance by eliminating the fetching of the data from the critical path of servicing the request and reduces query delay and improves the cache hit ratio. However, in case of wrong prediction, pre-fetching holds resources that could be used for other activities. In general pre-fetching systems use heuristics to balance these concerns and decide when and how much data to pre-fetch.

\subsection{Index caching Approach}

Index caching approach improves the query response time at the central server and since the different size of cache in mobile client affects the access time, thus this approach must be analyzed to minimize the response time subject to power consumption constraint. Moreover this approach may reduce the cost of maintaining mobile database for the mobile client.

\subsection{Judicious Caching}

Judicious Caching is used in case of frequent access of central server by the clients of a cell. Thus a copy of cache is maintained at the local server and keeps them always up to date. In this way, the clients in the cell can always access fresh copies of the data item directly from the local server without further delay. However, to keep the cached copies up to date, a local server must spend the extra cost of maintenance of getting new copies from the central server whenever updates occur.

\section{SIMULATION FRAMEWORK DESIGN TO STUDY THE COMMUNICATION COST OF CACHE APPROACHES}

The simulation framework has been studied with five cells and six mobile users to study the communication cost of three caching techniques pre-fetching, index caching and judicious caching. Each cell holds a local server as wireless information server to directly communicate with all mobile client for that particular cell. All the five local servers are connected to the central server and acts as bridge between the central server and the client devices at the same time. It is the center for managing local information as well as the key player to provide location based services. This is an important factor since neighboring local servers must work closely together to provide efficient location-based services. The cache and data manager of the local server is responsible for maintaining the information received from the central server as well as other local servers.

The mobile client simply can send out information requests via a wireless link, can do some local processing if required, and has a cache for keeping frequently accessed data items. Since user is mobile, it can easily change its location from one cell to another cell without the obligation to notify any server in advance. Since a mobile client can issue a query at any time anywhere, this is especially challenging for information service providers. In this architectural design, a client always sends requests to the local server of the cell where the user resides. The query object which is targeted may be available at the mobile cache, at the local server of the same cell region, from the local servers of other cells, or from central servers.

This effective cost model facilitate the design of dynamic data management of the location aware services that abstract away nonessential details of the wireless environment and characterize only the necessary objects required and prevailing cost factors that impact on the communication cost. As the $\mathrm{m}$ - commerce system must contain the mobile application, mobile station, wireless network, wired network and host computers. Thus to evaluate the communication cost to access the location based service three factors $\left(\mathrm{U}_{\mathrm{C}}, \mathrm{Tn}, \mathrm{Nb}\right)$ are considered. Here $\mathrm{U}_{\mathrm{C}}, \mathrm{Tn}$ and $\mathrm{Nb}$ are:

$\mathrm{U}_{\mathrm{C}}$-The unit cost of communication system

$\mathrm{Tn}$ - Total number of feasible connections

$\mathrm{Nb}$ - Average no. of bytes of each data item

The user access the network through a wireless connection to the local server while all other connections between servers (local and/or central) are through wired links. As the current technology stands, the cost of a wireless link is much lower than that of a wired link. The connection cost between neighboring servers is relatively lower than the cost of accessing from a remote central server. We therefore classify the communication cost into the following categories:

\begin{tabular}{|l|l|}
\hline Tc & Total Communication Cost \\
\hline Td & $\begin{array}{l}\text { Total Communication Cost for Database } \\
\text { Maintenance }\end{array}$ \\
\hline Tcq & $\begin{array}{l}\text { Total Communication Cost for querying and } \\
\text { accessing data items }\end{array}$ \\
\hline Qc & Query Cost for Mobile Customer \\
\hline $\mathbf{C w}$ & $\begin{array}{l}\text { Communication Cost between Local Server and } \\
\text { Mobile Client }\end{array}$ \\
\hline $\mathbf{C w l}$ & $\begin{array}{l}\text { Communication Cost between Local Server and } \\
\text { other neighbor Local Server }\end{array}$ \\
\hline $\mathbf{C w s}$ & $\begin{array}{l}\text { Communication Cost between Local Server and } \\
\text { Central Server }\end{array}$ \\
\hline $\mathbf{A h r}$ & Average Hit ratio of Mobile Client Cache \\
\hline $\mathbf{P m c}$ & $\begin{array}{l}\text { Probability of a Mobile Customer accessing data } \\
\text { item }\end{array}$ \\
\hline $\mathbf{M m c}$ & Mobility of Mobile Client \\
\hline $\mathbf{V c}$ & $\begin{array}{l}\text { Variation of Unit Cost of the Communication in } \\
\text { the System }\end{array}$ \\
\hline
\end{tabular}

Thus the total communication cost has been analyzed with the help of the factors ( $\mathrm{Uc} \mathrm{Tn}, \mathrm{Nb}$ ) as

$$
\mathrm{Tc}=\mathrm{Td}+\mathrm{Tcq}=\mathrm{T}(\mathrm{Uc}, \mathrm{Tn}, \mathrm{Nb})
$$

Here Td is total communication cost for database maintenance and Tcq is total communication cost for querying and accessing data items.

Due to replication and caching the total database maintenance cost $(\mathrm{Td})$ consists the cost of cache replacement and cache invalidation method. The cache replacement required due to frequent changing data size and data update in limited space for storage of data. Further cache invalidation mechanism 
ensures the consistency between data of the mobile client cache and data of the server[10].

The communication cost for querying and accessing the data item includes communication cost for sending out the request by mobile client, the communication cost of establishing a connection between the mobile client and local server and communication cost after connection is established.

i.e $\mathrm{Tcq}=\mathrm{Qc} . \mathrm{Ahr}+\mathrm{Cw} \cdot(1-\mathrm{Ahr})+\mathrm{Cwl}+\mathrm{Cws}$

Thus the total communication cost can be further explored as

$\mathrm{Tc}=\mathrm{Td}+\mathrm{Qc} \cdot \mathrm{Ahr}+\mathrm{Cw} \cdot(1-\mathrm{Ahr})+\mathrm{Cwl}+\mathrm{Cws}$

Consider the communication cost for querying and accessing the data items, where the factors $\mathrm{Qc}, \mathrm{Cw}, \mathrm{Cws}$, Cwl for the three considered caches techniques varies as the values of the three factors Pmc, Mmc and $\mathrm{Vc}$ changes and $\mathrm{Ahr}$ remain constant. Thus, this simulation study does the analysis that Access Probability (Pmc), Mobility (Mnc), cache Hit Ratio (Ahr) affects the Total Communication Cost. At present Hit Ratio (Ahr) is kept constant.

Let $\mathrm{P}$ be probability to access the data item then 1-P the non-access probability

Table 1: The values of parameters for simulation study.

\begin{tabular}{|l|c|c|c|c|}
\hline $\begin{array}{l}\text { Caching } \\
\text { techniques }\end{array}$ & $\begin{array}{l}\text { Query } \\
\text { Cost( } \\
\text { Qc) }\end{array}$ & $\begin{array}{l}\text { Wireless } \\
\text { Communica } \\
\text { tion Cost } \\
\text { MC to } \\
\text { LS(Cw) }\end{array}$ & $\begin{array}{l}\text { Cabl } \\
\text { e } \\
\text { Cost } \\
\text { LS- } \\
\text { to } \\
\text { LS(C } \\
\text { wl) }\end{array}$ & $\begin{array}{l}\text { Local } \\
\text { to } \\
\text { centr } \\
\text { al } \\
\text { serve } \\
\text { r } \\
\text { cost( } \\
\text { Cws) }\end{array}$ \\
\hline $\begin{array}{l}\text { Index } \\
\text { Caching(*Rs./Q } \\
\text { uery) }\end{array}$ & 10 & 20 & 100 & 500 \\
\hline $\begin{array}{l}\text { Judicious } \\
\text { Caching(*Rs./Ti } \\
\text { me Unit) }\end{array}$ & 5 & 20 & 100 & 10 \\
\hline $\begin{array}{l}\text { Precaching(*Rs. } \\
\text { /Occ.) }\end{array}$ & 1 & 10 & 50 & 20 \\
\hline
\end{tabular}

*Rs is considered as currency

In the present paper, simulation is based upon the graphical representation of relationship between various parameters with the initial behaviour of the mobile client and access the mobile data base for different utility and movement among different cells (we have considered five cells for mobility point of view). The effort has also been done to simulate among three different types of caching approaches. The use of these caching approaches are based upon the access to the mobile database and the relationship among the two varing parameters: access probability, mobility, and the third parameter hit ratio is kept constant.

\section{Mobile Client Acccess behavior:}

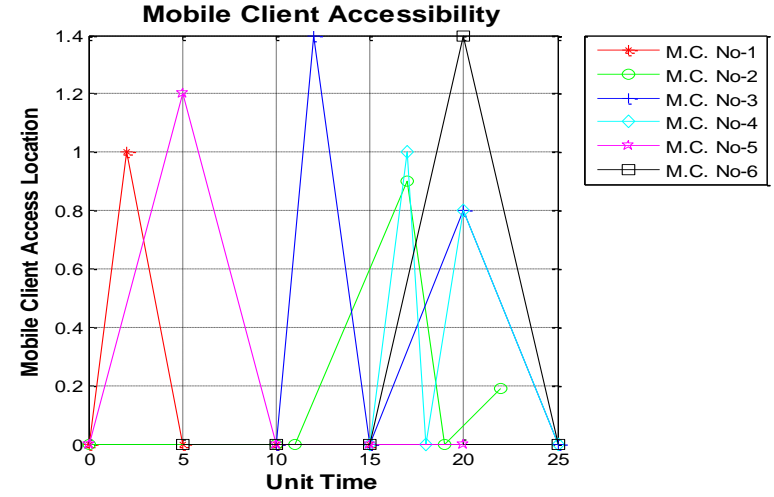

Fig 1: Mobile Client Access Probability $\mathbf{p}=\mathbf{0 . 0 5}$

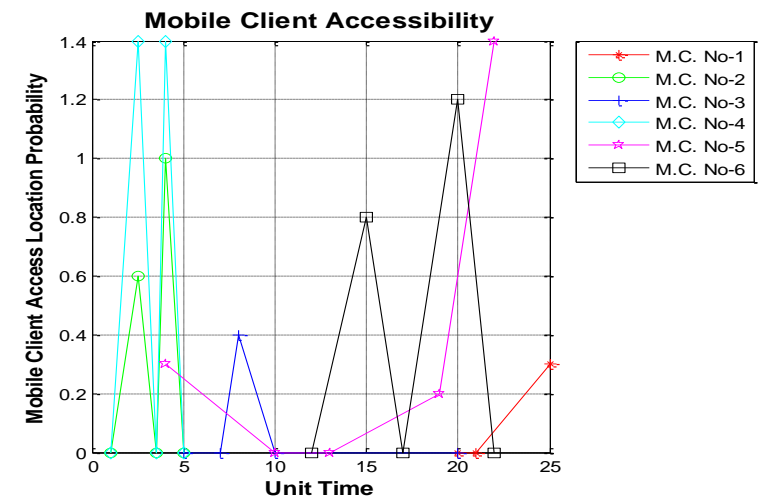

Fig 2: Mobile Client Access Probability with $\mathrm{p}=\mathbf{0 . 2 5}$

Basically we have considered two probability to study the behaviour of mobile clients to the mobile data base say: $\mathrm{P}=.05$ and .25. The mobile client uses cache randomly from one of the local database and central database more frequently in case $\mathrm{p}=.25$, the mobile client number two and four access the cell 1 more frequently means the access of the cache is very high and fast. These two clients feches the data from local and central database server more frequently. The other client access is occassionaly based on the data need time to time.

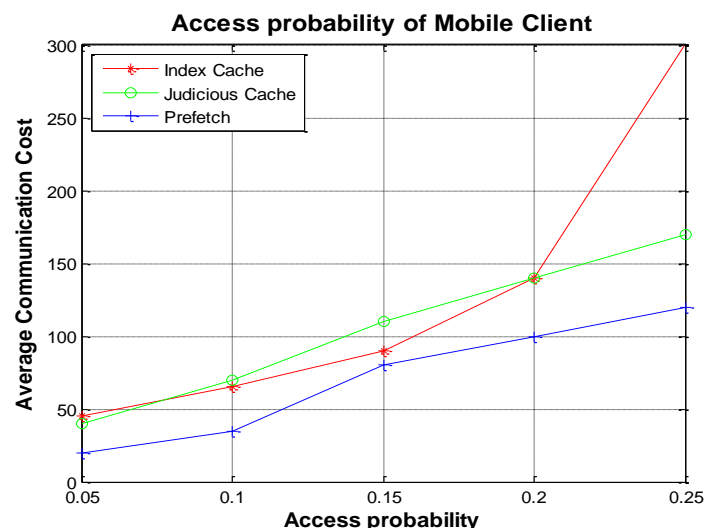

Fig 3: Total Communication Cost vs. Access Probability

Two parameters were variable and the third parameter hit ratio to be .35 and .40 . As the mobile user access increases from .05 to onward. Total communication cost rises w.r.t. rate of mobility. Moreover in case of Index caching, the simulation result shows an exponential change beyond access probability 
more than 0.2 . The rate of growth of perfecting is normal with the rise of access probability.

\section{Mobile Client Mobility Behavior:}

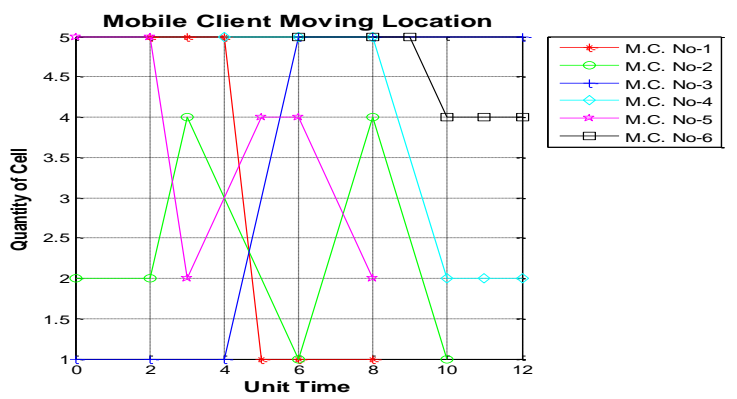

Fig 4: The Mobile Client Mobility

The graph show the behavior of mobile client w.r.t. inter and intra mobility for analysis quality of cells. In case the mobility is high in a cell, it is due to drop of the call or requisite information is not available in cache and searches from local server. In case of local to local or local to central server, the mobility user leaves the cell and fetches the data from another cell.

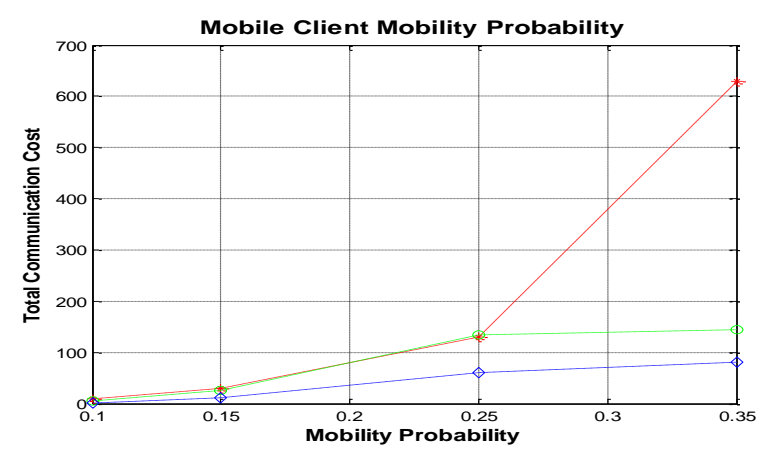

Fig 5: Total Communication Cost vs Mobility

\section{CONCLUSION:}

Mobile Commerce (M-Commerce) is the access of information from different locations by the mobile clients. Mcommerce saves the time and other transaction efforts among sellers and buyers. Communication cost depends on wired, wireless cost of mobility of mobile client and frequent disconnection which increases the battery consumption and other maintenance cost. Frequent mobility from one cell to other, hit ratio, probability of access the data from Local data server, central server increases the communication cost. To make the M-commerce services more attractive, the communication cost should be reduced. Through frame work of this paper, an effort has been done by the authors to find out the ways and means to reduce the communication cost and further suggesting that how M-commerce can be made more attractive. As replicating the data through various approaches like index caching, judicious caching and pre-fetching by mobile clients, reduces the communication cost for accessing the desired services. A simulation study has been done by the authors. The simulation study is based on three caching approaches and two varying parameters: access probability and mobility either in the same location or one location to other, though one more parameter i.e. Hit-Ratio which also affects the communication cost is kept constant in the present study. Only one time Hit-Ratio is taken into account. The access probability and mobility of the user from one location to another location for fetching the information more frequently which increases the cost accordingly. Fig.1 \&2, depict that if the access probability increase, the mobile client fetch the data from Local data server from same location or go to other locations, a number of times and if data is available, there is no further hunting or access otherwise the client approaches the central server. If this access probability further increases, the communication cost increases exponentially as shown in Fig. 3 . Moreover, the behavior of the mobile user during M-commerce applications also invites call drop and increases the access rate, hit ratio which also effects the total communication cost. Therefore, the communication cost can be reduced at an appreciable rate, if access probability and mobility of the clients is kept limited.

\section{REFERENCES}

[1] Anckar B and D'Incau D(2002) 'Value creation in mobile commerce: Findings from a c onsumer survey.' . The Journal of Information Technology Theory and Application (JITTA) 4 ( 1): 43-64

[2] Steinfield C, Bouwman H and Adelaar T(2002) 'The dynamics of click and mortar e-commerce: Opportunities and management strategies.' International Journal of Electronic Commerce 7(1): 93-119

[3] D'Roza T and and Bilchev G (2003) 'An overview of location-based services.' BT Technology Journal 21(1): 20-27.

[4] Brewin R (2001) 'Merchant Wired rolling out 'wireless' mall for retailers.' Computerworld May 30.

[5] Haroon Shahzad, Xiang Li and Muhammad Irfan (2013) Review of Data Replication Techniques for Mobile Computing Environment' Research Journal of Applied Sciences, Engineering and Technology 6(9): 1639-1648, 2013 ISSN: 2040-7459; e-ISSN: 2040-7467 .

[6] I.-d. Jung, Y.-h. You, J.-h. Lee, and K. Kim(2002) 'Broadcasting and caching policies for locationdependent queries in urban areas.' ACM, 2002, ISSN 2047-3338, pp. 54-60.

[7] Kshama Raichura,Nilesh Padhariyaand Kishor tkotiya(2013) International Journal of Computer Science and Telecommunications, Volume 4, Issue 5.

[8] Daniel Barbara(1999) 'Mobile Computing and Databases -a servey' IEEE transactions on Knowledge and Data Engineering,11(1): 108-117,

[9] Michael J. Franklin, Michael J. Carey, and Miron Livny(1992) 'Global memory management in clientserver database architectures'. In VLDB '92: Proceedings of the 18th International Conference on Very Large Data Bases, pages 596-609, San Francisco, CA, USA,

[10] Baihua Zheng, Jianliang Xu and Dik lee(2002) ' Cache Invalidation \& Replacement Strategies for Location dependent Data in Mobile Environment: IEEE Transaction on Computers, pp 1141-1153. 